\title{
BIBLIOGRAFÍA CRÍTICA SOBRE FRANCISCO DE ROJAS ZORRILLA
}

RAFAEL GONZÁLEZ CAÑAL Universidad de Castilla-La Mancha

Hace ya más de cuatro años elaboramos una primera bibliografía crítica sobre Francisco de Rojas Zorrilla (González Cañal [2003]). Al celebrar ahora el año del centenario del dramaturgo toledano conviene actualizar aquel repertorio bibliográfico antes de asistir a la oleada de ediciones y estudios que se anuncian para próximas fechas.

Mucho se ha avanzado en los últimos años en los estudios sobre el teatro de este poeta dramático, desde aquel trabajo señero de Cotarelo [1911] y los posteriores de Raymond R. MacCurdy, que siguen siendo referencias inexcusables. Las XXII Jornadas de teatro clásico de Almagro, que se celebraron en 1999, supusieron el punto de partida para profundizar en el análisis y estudio de la obra de este dramaturgo. Las actas de aquel encuentro, publicadas al año siguiente (Pedraza Jiménez, González Cañal y Marcello [2000]), contienen algunas de las aportaciones críticas más relevantes del último período. A ellas hay que añadir los numerosos trabajos sobre aspectos concretos que han ido sacando a la luz investigadores como Felipe B. Pedraza Jiménez o María Teresa Julio. El primero de ellos acaba de reunir en un volumen sus estudios sobre Rojas publicados en estos últimos años. Contamos también ahora con un instrumento fundamental como es la bibliografía individual del toledano, en donde se recogen y describen con criterios rigurosos todas las ediciones de cada una de sus obras (González Cañal, Cerezo Rubio y Vega García-Luengos [2007]).

En esta bibliografía crítica recopilamos las ediciones y estudios más importantes sobre la vida y la obra de Rojas. Evidentemente, en cualquier repertorio de este tipo se producen olvidos y errores, y debe ser continuamente actualizado con las nuevas aportaciones que van surgiendo. Sirva de momento esta bibliografía de herramienta y ayuda para los estudios sobre Rojas Zorrilla que están en curso. 


\section{EDICIONES}

Primera parte de las comedias de Don Francisco de Rojas de Zorrilla, Madrid: María de Quiñones. A costa de Pedro Coello, Mercader de libros, 1640. Contiene las siguientes comedias: No hay amigo para amigo; No hay ser padre siendo rey; Donde hay agravios no hay celos; Casarse por vengarse; Obligados y ofendidos; Persiles y Segismunda; Peligrar en los remedios; Los celos de Rodamonte; Santa Isabel, reina de Portugal; La traición busca el castigo; El profeta falso Mahoma; Progne y Filomena.

Segunda parte de las comedias de Don Francisco de Rojas Zorrilla, Madrid: Francisco Martínez. A costa de Pedro Coello, Mercader de libros, 1645: Lo que son mujeres; Los bandos de Verona; Entre bobos anda el juego; Sin honra no hay amistad; Nuestra Señora de Atocha; Abrir el ojo; Los trabajos de Tobías; Los encantos de Medea; Los tres blasones de España (en colaboración con Antonio Coello); Los áspides de Cleopatra; Lo que quería ver el marqués de Villena; El más impropio verdugo.

Parte primera de las comedias de don Francisco de Roxas Zorrilla, Madrid: Lorenzo García de la Iglesia, 1680.

Parte segunda de las comedias de don Francisco de Roxas Zorrilla... Madrid: Lorenzo de la Iglesia, 1680.

Comedias escogidas de don Francisco de Rojas Zorrilla, Madrid, Ortega y Compañía, 1827-31, 2 vols. Tomo I: Del rey abajo ninguno (pp. 3-110); Donde hay agravios no hay celos (pp. 111-256); Entre bobos anda el juego (pp. 257-396); Don Diego de noche (pp. 397-641); Índice (p. 642).Tomo II: Lo que son mujeres (pp. 1-142); Abre el ojo (pp. 143-287); El desdén vengado (pp. 289-399); Progne y Filomena (pp. 401-557).

Tesoro del teatro español desde su origen (año de 1356) hasta nuestros días, ed. Eugenio de Ochoa, tomo 4, París, Librería Europea de Baudry (Imprenta de Casimir), 1838 (tomo 13 de la Colección de los mejores autores españoles): Del rey abajo, ninguno (pp. 339-366), Donde hay agravios no hay celos (pp. 366-400) y Entre bobos anda el juego (pp. 400-431).

Francisco DE Rojas ZoRrilla, Comedias escogidas..., ed. Ramón Mesonero Romanos, Madrid, M. Rivadeneyra, 1861; Madrid, Atlas, 1952. (BAE, 54). Eds.: 1861, 1866, 1897, 1908, 1918, 1926 y 1952.

Tesoro selecto antiguo y moderno, nacional y extranjero, coleccionado e ilustrado con una introducción, notas, observaciones críticas y biografías de los principales autores, por Don Francisco José Orellana, tomo II, Barcelona, Salvador Manero, 1867: Del rey abajo ninguno y labrador más honrado García del Castañar (pp. 325-350); Don Diego de noche (pp. 351-380); Donde hay agravios no hay celos, y amo criado (pp. 381-412); Obligados y ofendidos y gorrón de Salamanca (pp. 413-446); Entre bobos anda el juego, don Lucas del Cigarral (pp. 447-476); La traición busca el castigo (pp. 477-510); Lo que son mujeres (pp. 511-540); y Abre el ojo (pp. 541570).

Comedias escogidas de Francisco de Rojas Zorrilla..., Barcelona, Daniel Cortezo y Compañía, 1884 (Biblioteca Clásica Española): Del rey abajo ninguno y labrador más honrado García del Castañar (pp. 7-78); Entre bobos anda el juego, D. Lucas del Cigarral (pp. 79-163); Lo que son mujeres (pp. 165-252); y Donde hay agravios, no hay celos, y amo criado (pp. 253-344).

Francisco DE Rojas Zorrilla, Obras completas, I, ed. del Instituto Almagro de teatro clásico, dirigida por Felipe B. Pedraza Jiménez y Rafael González Cañal, Cuenca, Universidad de Castilla-La Mancha, 2007: No hay amigo para amigo (ed. Rafael 
González Cañal), No hay ser padre siendo rey (ed. Enrico di Pastena), Donde hay agravios, no hay celos (ed. Felipe B. Pedraza y Milagros Rodríguez Cáceres) y Casarse por vengarse (ed. María Teresa Julio).

- Obras completas, II, ed. del Instituto Almagro de teatro clásico, dirigida por Felipe B. Pedraza Jiménez y Rafael González Cañal, Cuenca, Universidad de Castilla-La Mancha, en prensa: Obligados y ofendidos, Persiles y Segismunda, Peligrar en los remedios y Los celos de Rodamonte.

Cada cual lo que le toca. La viña de Nabot, ed. Américo Castro, Madrid, Sucesores de Hernando, 1917.

Del rey abajo, ninguno. Entre bobos anda el juego, ed. Federico Ruiz Morcuende, Madrid, Ediciones de «La Lectura», 1917; 5a edición: Madrid, Espasa-Calpe (col. Clásicos Castellanos, 35), 1967.

Del rey abajo ninguno, ed. Pablo Pou Fernández, Zaragoza, Clásicos Ebro, 1964.

Del rey abajo, ninguno o El labrador más honrado García del Castañar, ed. Jean Testas, Madrid, Castalia, 1971.

Del rey abajo, ninguno, ed. Brigitte Wittmann, Madrid, Cátedra (col. Letras Hispánicas, 132), 1980.

Del rey abajo, ninguno. Entre bobos anda el juego, ed. Ana Suárez Miramón, Barcelona, Planeta (col. Autores Hispánicos, 182), 1990.

Donde ay agravios no ay zelos, ed. Brigitte Wittmann, Ginebra-París, E. Droz-Minard, 1962.

Donde hay agravios no hay celos y Abrir el ojo, ed. Felipe B. Pedraza Jiménez y Milagros Rodríguez Cáceres, Madrid, Castalia (col. Clásicos Castalia, 282), 2005.

Don Gil de la Mancha, en Paul Ferdinand Luenow, An Edition of "Don Gil de la Mancha», Tesis doctoral inédita, Albuquerque, The University of New Mexico, 1955. Entre bobos anda el juego, ed. Maria Grazia Profeti, Madrid, Taurus, 1984.

Entre bobos anda el juego, ed. Maria Grazia Profeti, Barcelona, Crítica, 1998.

El jardín de Falerina, en Robert R. Bacalski, A Critical Edition of «El jardín de Falerina» by Rojas Zorrilla, Antonio Coello and Calderón, Tesis doctoral, Albuquerque, The University of New Mexico, 1971.

Lucrecia y Tarquino, ed. Raymond R. MacCurdy, Alburquerque/New Mexico, Universidad de New Mexico Press, 1963.

El mejor amigo, el muerto, en colaboración con Luis Belmonte Bermúdez y Pedro Calderón de la Barca, en Pedro Calderón de la Barca, Comedias, IV, ed. Juan Eugenio Hartzenbusch, Madrid, Rivadeneyra, 1850 (BAE, 14).

El monstruo de la fortuna. La lavandera de Nápoles, Felipa de Catanea, en colaboración con Pedro Calderón de la Barca y Juan Pérez de Montalbán, edizione critica, introducione e note a cura di Germana Volpe, Napoli, Università degli Studi di Napoli «L'Orientale», 2006.

Morir pensando matar. La vida en el ataúd, ed. Raymond R. MacCurdy, Madrid, Espasa Calpe (col. Clásicos Castellanos, 153), 1961.

Nuestra Señora de Atocha, en Kevin Thomas Schemelter, An annotated critical edition of Francisco de Rojas Zorrilla's «Nuestra Señora de Atocha», Tesis doctoral, Albuquerque, The University of New Mexico, 1969.

Numancia cercada y Numancia destruida, ed. Raymond R. MacCurdy, Madrid, José Porrúa Turanzas, 1977.

Obligados y ofendidos, ed. Patricia Petersen. Albuquerque, New Mexico, 1952 (Tesis M.A. Univ. New Mexico).

Obligados y ofendidos, ed. Raymond R. MacCurdy, Salamanca, Anaya, 1963. 
Progne y Filomena, ed. Alfredo Rodríguez y Saúl E. Roll Vélez, New York, Peter Lang, 1994.

También tiene el sol menguante, en James S. Rambo, An Annotated Critical Edition of «También tiene el sol menguante» by Luis Vélez and Rojas Zorrilla, Tesis doctoral, Albuquerque, The University of New Mexico, 1972.

Los trabajos de Tobías, en Harriet B. Powers, A critical edition of Francisco e Rojas Zorrilla's «Los trabajos de Tobías», Tesis doctoral, Albuquerque, The University of New Mexico, 1967.

Los tres blasones de España, en colaboración con Antonio Coello, ed. Ana Belén Fernández Urenda, Francisco Javier Fernández Urenda y María Corral Lumbreras, Calahorra, Ayuntamiento de, Calahorra, 1998. Reimpresión, con distinta distribución de páginas: Ayuntamiento de Calahorra, 1999.

\section{ESTUDIOS}

Álvarez Sellers, María Rosa [1997]: «Morir pensando matar (1642). Francisco de Rojas Zorrilla», en Análisis y evolución de la tragedia española en el Siglo de Oro. La tragedia amorosa, Kassel, Reichenberger, III, pp. 805-824.

AREllano AyUSO, Ignacio [1993]: «Rojas Zorrilla», en Historia de la literatura española II. Renacimiento. Barroco, coord. Jesús Menéndez Peláez, León, Everest, pp. 466-475.

- [1995]: «Rojas Zorrilla», en Historia del teatro español del siglo XVII, Madrid, Cátedra, pp. 549-577.

- [2001]: «Rojas Zorrilla», en Calderón y su escuela dramática, Madrid, Ediciones del Laberinto (colecc. «Arcadia de las Letras»), pp. 128-141.

ARENAS CRUZ, María Elena [2000]: «Las representaciones de Rojas en el siglo XVIII y su valoración en el Memorial literario», en Francisco de Rojas Zorrilla, poeta dramático. Actas de las XXII Jornadas de teatro clásico, Almagro 13, 14 y 15 de julio de 1999, ed. Felipe B. Pedraza Jiménez, Rafael González Cañal y Elena Marcello, Almagro (Ciudad Real), Universidad de Castilla-La Mancha-Festival de Almagro, pp. 379-394.

- [2004]: «Francisco de Rojas Zorrilla», en Paraninfos, segundones y epígonos de la comedia del Siglo de Oro, coord. Ignacio Arellano, Barcelona, Anthropos-GRISO, 2004, pp. 157-163.

- [2005]: «Imaginarios campos de batalla en las obras de Rojas Zorrilla», en Espacio, tiempo y género en la comedia española. Actas de las II Jornadas de teatro clásico. Toledo, 14, 15 y 16 de noviembre de 2003, ed. Felipe B. Pedraza Jiménez, Rafael González Cañal y Gemma Gómez Rubio, Almagro, Universidad de Castilla-La Mancha, pp. 247-264.

ARMAS, Frederick A. de [1989]: «En Madrid y en un casa: un palimpsesto de amantes invisibles», en Actas del IX Congreso de la Asociación Internacional de Hispanistas, publicadas por Sebastian Neumeister, Frankfurt am Main, Vervuert Verlag, I, pp. 341-351.

- [2000]: «Numancia as Ganymede: Conquest and continence in Giulio Romano, Cervantes and Rojas Zorrilla», en Echoes and Inscriptions: Comparative Approaches to Early Modern Spanish Literatures, ed. Barbara Simerka y Christopher B. Weimer, Lewisburg, PA, Bucknell UP, pp. 250-270.

AZCunE, Valentín [2000]: «Miscelánea erudita», en Cuadernos para la Investigación de la Literatura Hispánica, n. ${ }^{\circ}$ 25, pp. 267-280. 
BAllesteros GonZÁlez, Antonio [2000]: «Vaporosas simetrías: la huella de Rojas Zorrilla en William Davenant», en Francisco de Rojas Zorrilla, poeta dramático. Actas de las XXII Jornadas de teatro clásico, Almagro 13, 14 y 15 de julio de 1999, ed. Felipe B. Pedraza Jiménez, Rafael González Cañal y Elena Marcello, Almagro (Ciudad Real), Universidad de Castilla-La Mancha-Festival de Almagro, pp. 303-322. BARRET, John Alfred [1938]: Some Aspects of the Dramatics Techniques of Francisco de Rojas Zorrilla, Chapel Hill, Tesis M. A., University of North Carolina.

Blue, William R. [1994]: «The diverse economy of Entre bobos anda el juego», en The Golden Age Comedia: Text, Theory ab Performance, ed. Charles Ganelin y Howard Mancing, West Lafayette, Purdue University Press, pp. 76-86.

Boletín de la Compañía Nacional de Teatro Clásico, núm. 40, 1999 (dedicado a Entre bobos anda el juego).

Boorman, John T. [1950]: The dramatic technique of Rojas Zorrilla, Tesis doctoral inédita, Leeds College.

Bravo Carbonell, J. [1908]: El toledano Rojas, Toledo, Tipografía de Rafael GómezMenor.

BRIESEMEISTER, D. [1983]: «El horror y su función en algunas tragedias de Rojas Zorrilla», en Criticón, 23, pp. 159-175. Fragmentos reproducidos en Francisco Rico, dir., Historia y crítica de la Literatura Española, 3/1, Siglos de Oro: Barroco. Primer suplemento, Barcelona, Crítica, 1992, pp. 483-487.

Brioso SANTOS, Héctor [2000]: «Nuevas consideraciones acerca del final de Entre bobos anda el juego de Francisco de Rojas Zorrilla: la estructura de la comedia y las convenciones del género», en Hesperia (Vigo), III, pp. 5-23.

- [2000]: «Comentarios al desenlace de Entre bobos anda el juego de Francisco de Rojas Zorrilla», en Castilla. Estudios de Literatura, 25, pp. 43-59.

- [2001]: «Rasgos entremesiles de la comedia de figurón: Rojas Zorrilla y Matos Fragoso», en Philologia Hispalensis, 15, pp. 23-31.

BUSQUETS, Loreto [1991]: «Lucrecia y Tarquino o el conflicto entre el fin y los medios», en Nueva Revista de Filología Hispánica, XXXIX, pp. 977-1004.

CALDERA, Ermano [1962]: «Solitudine dei personaggi di Rojas», en Studi Ispanici (Universidad de Pisa), I, pp. 37-60.

CANo NAVARro, José [2003]: «Autoría de La difunta pleiteada: )Rojas Zorrilla o Lope de Vega?», en Con Alonso Zamora Vicente. (Actas del Congreso Internacional «La lengua, la Academia, lo popular, los clásicos, los contemporáneos...»), Murcia, Universidad de Alicante, II, pp. 465-475.

CAÑAS Murillo, Jesús [1981]: «Unidad en la dualidad: La segunda acción en Cada cual lo que le toca, de Rojas Zorrilla», en Anuario de Estudios Filológicos (Cáceres), IV, pp. 39-54.

CASTILlA, Alberto [1986]: «Seis autores en busca de una actriz: la Baltasara», en Actas del VIII Congreso de la Asociación Internacional de Hispanistas, Brown University, 22-27 de agosto 1983, ed. A. David Kossoff et al., Madrid, Eds. Istmo, I, pp. 367-380.

CASTILlejo, David [2002]: «Francisco de Rojas Zorrilla (1607-1648)», en Guía de Ochocientas Comedias del Siglo de Oro para el uso de actores y lectores, Madrid, Ars Milenii, pp. 635-656.

CAStro, Américo [1906]: «Obras mal atribuidas a Rojas Zorrilla», en Revista de Filología Española, III, pp. 66-68.

Cattaneo, María Teresa [1992]: «Medea, entre mito y magia. En torno a la comedia de magia de Rojas Zorrilla», en La comedia de magia y de santos, Madrid, Júcar, pp. 123-131.

- [2000]: «Los desenlaces en el teatro de Rojas Zorrilla», en Francisco de Rojas Zorrilla, poeta dramático. Actas de las XXII Jornadas de teatro clásico, Almagro 
13, 14 y 15 de julio de 1999, ed. Felipe B. Pedraza Jiménez, Rafael González Cañal y Elena Marcello, Almagro (Ciudad Real), Universidad de Castilla-La ManchaFestival de Almagro, pp. 39-53.

CONLON, Raymond [1988]: «Animal Symbolism and the characterization of Mendo in Rojas Zorrilla's Del rey abajo, ninguno», en Bulletin of the Comediantes (Toronto), XL, pp. 13-23.

- [1990]: «Mendo as social enemy in Rojas Zorrilla's Del Rey abajo, ninguno», en Crítica Hispánica (Johnson City), XII, n. ${ }^{\circ}$ 1-2, pp. 65-74.

Cotarelo y Mori, Emilio [1911]: Don Francisco de Rojas Zorrilla, noticias biográficas y bibliográficas, Madrid, Imprenta de la Revista de Archivos.

COUDERC, Christophe [2000]: «Recepción y adaptación de Rojas Zorrilla en Francia (siglo XVII): algunos ejemplos», en Francisco de Rojas Zorrilla, poeta dramático. Actas de las XXII Jornadas de teatro clásico, Almagro 13, 14 y 15 de julio de 1999, ed. Felipe B. Pedraza Jiménez, Rafael González Cañal y Elena Marcello, Almagro (Ciudad Real), Universidad de Castilla-La Mancha-Festival de Almagro, pp. 323-348.

CRUICKSHANK, D. W. [1977]: «Rojas Zorrilla's Lucrecia y Tarquino: The date and printer of the first known edition», en Modern Language Notes, XCII, n. ${ }^{\circ}$ 2, pp. 329-331.

CRuz CASAdO, Antonio [1993]: «Persiles y Sigismunda. De Cervantes a Rojas Zorrilla», en Actas del Tercer Coloquio Internacional de la Asociación de Cervantistas. Alcalá de Henares, 12-16 nov. 1990, Barcelona, Ministerio de Asuntos Exteriores- Anthropos, pp. 541-551.

DÉODAT-KESSEDJIAN, Marie-Françoise [2000]: «Las obras escritas en colaboración por Rojas Zorrilla y Calderón», en Francisco de Rojas Zorrilla, poeta dramático. Actas de las XXII Jornadas de teatro clásico, Almagro 13, 14 y 15 de julio de 1999, ed. Felipe B. Pedraza Jiménez, Rafael González Cañal y Elena Marcello, Almagro (Ciudad Real), Universidad de Castilla-La Mancha-Festival de Almagro, pp. 209-239.

- [2005]: «El concepto de santidad en la comedia áurea: algunos ejemplos dispares de 'santas'», en Homenaje a Henri Guerreiro. La hagiografía entre historia y literatura en la España de la Edad Media y del Siglo de Oro, ed. Marc Vitse, Pamplona, Universidad de Navarra-Iberoamericana Vervuert, pp. 493-509.

DOMÉNECH RiCO, Fernando [1999]: «Análisis de Obligados y ofendidos», en F. de Rojas Zorrilla, Obligados y ofendidos, Madrid, RESAD-Ed. Fundamentos, pp. 69-130.

- [2000]: «Los bandos de Verona, comedia áulica», en Francisco de Rojas Zorrilla, poeta dramático. Actas de las XXII Jornadas de teatro clásico, Almagro 13, 14 y 15 de julio de 1999, ed. Felipe B. Pedraza Jiménez, Rafael González Cañal y Elena Marcello, Almagro (Ciudad Real), Universidad de Castilla-La Mancha-Festival de Almagro, pp. 151-178.

EIROA, Sofía [2000]: «Las protagonistas femeninas de Rojas Zorrilla: Lo que son mujeres», en Francisco de Rojas Zorrilla, poeta dramático. Actas de las XXII Jornadas de teatro clásico, Almagro 13, 14 y 15 de julio de 1999, ed. Felipe B. Pedraza Jiménez, Rafael González Cañal y Elena Marcello, Almagro (Ciudad Real), Universidad de Castilla-La Mancha-Festival de Almagro, pp. 121-132.

Elejabeitia, Ana [1991]: «La transmisión textual de La más hidalga hermosura», en Letras de Deusto, XXI, n. ${ }^{\circ}$ 51, pp. 53-65.

ENTRAmBASAguAS, Joaquín de [1948]: «La leyenda de Rosamunda», en Revista Bibliográfica y Documental (Madrid), II, pp. 339-389; recogido en Estudios y ensayos de investigación y crítica. De la leyenda de Rosamunda a Jovellanos, Madrid, CSIC, 1973, pp. 3-54 (Sobre las fuentes de Morir pensando matar, de Rojas).

ESTEFANÍA, Dulce [2001]: «Francisco de Rojas Zorrilla y Agustín Moreto. Dos ejemplos de tratamiento de la mitología», en Myrtia, 16, pp. 259-274.

FARRELl, Anthony J. [2005]: «Tiempo y espacio en Lo que quería ver el marqués de Villena», en Espacio, tiempo y género en la comedia española. Actas de las II Jor- 
nadas de teatro clásico. Toledo, 14, 15 y 16 de noviembre de 2003, ed. Felipe B. Pedraza Jiménez, Rafael González Cañal y Gemma Gómez Rubio, Almagro, Universidad de Castilla-La Mancha, pp. 103-111.

FERNÁNDEZ FERNÁNDEZ, Olga [2000]: «Las estructuras funcionales de la comedia de figurón: la función del figurón en Entre bobos anda el juego», en Francisco de Rojas Zorrilla, poeta dramático. Actas de las XXII Jornadas de teatro clásico, Almagro 13, 14 y 15 de julio de 1999, ed. Felipe B. Pedraza Jiménez, Rafael González Cañal y Elena Marcello, Almagro (Ciudad Real), Universidad de Castilla-La ManchaFestival de Almagro, pp. 133-150.

FORASTIERI-BRASCHI, Eduardo [1992]: «Del rey abajo, ninguno, y la invención venatoria del hostigamiento», en Scripta Philologica in honorem Juan M. Lope Blanch... III. Lingüística indoamericana y Estudios Literarios, coord. Elizabeth Luna Traull, México, Universidad Nacional Autónoma de México, pp. 189-201.

FRIEDMAN, Edward H. [1989]: «Romeo and Juliet as tragicomedy: Lope's Castelvines y Monteses and Rojas Zorrillas's Los bandos de Verona», en Bucknell Review. 'Comedias del Siglo de Oro' and Shakespeare (Lewisburg, Bucknell University Press/ London \& Toronto, Associated University Presses) XXXIII, n. ${ }^{\circ}$ 1, pp. 82-96.

FuCILla, Joseph G. [1951]: «Sobre las fuentes de Del rey abajo, ninguno», en Nueva Revista de Filología Hispánica, V, pp. 381-393.

GARCía GonZÁLEZ, Almudena [2007]: «La figura de Juan Sala Serrallonga en El catalán Serrallonga y bandos de Barcelona», en Locos, figurones y quijotes en el teatro de los Siglos de Oro. Actas selectas del XII Congreso de la Asociación Internacional de Teatro Español y Novohispano de los Siglos de Oro. Almagro, 15, 16 y 17 de julio de 2005, ed. Germán Vega García-Luengos y Rafael González Cañal, Almagro, Universidad de Castilla-La Mancha, pp. 195-206.

- [en prensa]: «Roque Guinart y otros bandoleros literarios del siglo XVII», en Actas del Congreso Internacional sobre «Cervantes y su tiempo», León, 2-5 noviembre de 2005.

GARrot, Juan Carlos [2006]: «Hagiographie, identité locale et identité nationale: Los tres blasones de España de Francisco de Rojas Zorrilla et Antonio Coello», en Saints en scène. Théâtre et hagiographie en Europe (XIVe-XVIIe siècles), Journée d'études organisée par Jean-Pierre Bordier, Juan Carlos Garrot et Pierre Pasquier, Tours, Centre d'Etudes Supérieures de la Renaissance, Université de Tours.

Givanel MAS, Juan [1945]: «Observaciones sugeridas por la lectura del drama de Coello, Rojas y Vélez El catalán Serrallonga y Vandos de Barcelona», en Boletín de la Academia de Buenas Letras (Barcelona), XVIII, pp. 159-192.

Glaser, Edward [1957]: «Santa Isabel, reina de Portugal, de Francisco de Rojas Zorrilla», en Estudios Hispano-portugueses: Relaciones literarias del Siglo de Oro, Valencia, Editorial Castalia, pp. 179-220. Reproducido en A. T. CARnEIRo [1964], «Santa Isabel, reina de Portugal, de F. de Rojas Zorrilla, e a noticia critica do Prof. E. Glaser», en Arquivo Coimbrâo, XIX-XX, pp. 335-425.

Goldman, Rachel [1976]: The Lucretia legend from livy to Rojas Zorrilla, Tesis doctoral inédita, The City University of New York.

GómeZ Rubio, Gemma [2003]: «La adaptación de Peligrar en los remedios de Rojas Zorrilla, según un manuscrito de compañía», en Con Alonso Zamora Vicente. (Actas del Congreso Internacional «La lengua, la Academia, lo popular, los clásicos, los contemporáneos...», ed. Carmen Alemany et al., Alicante, Univ. de Alicante, 2003, pp. 673-682.

- [2005]: «La configuración de la tragedia en Lucrecia y Tarquino», en Espacio, tiempo y género en la comedia española. Actas de las II Jornadas de teatro clásico. Toledo, 14, 15 y 16 de noviembre de 2003, ed. Felipe B. Pedraza Jiménez, Rafael 
González Cañal y Gemma Gómez Rubio, Almagro, Universidad de Castilla-La Mancha, pp. 433-451.

- [2005]: «Relaciones de hipertextualidad y modos de dramatización en el teatro de Rojas Zorrilla», en Actas del Congreso «El Siglo de Oro en el nuevo milenio», ed. Carlos Mata y Miguel Zugasti, Pamplona, EUNSA, I, pp. 805-816.

- [en prensa]: «Rojas Zorrilla ante el universo palatino: el caso de Morir pensando matar», Lectura y Signo, 2 (2007).

GonZÁLEZ, Cristina [1980]: «Sobre Del rey abajo ninguno», en Bulletin of the Comediantes (Toronto), XXXII, pp. 49-53.

GonZÁleZ CAÑAL, Rafael [1999]: «Temas cervantinos en el teatro de Rojas Zorrilla», en Anales cervantinos, XXXV, pp. 193-203.

- [2003]: «Bibliografía crítica sobre Francisco de Rojas Zorrilla», en Toledo: entre Calderón y Rojas. IV Centenario del nacimiento de don Pedro Calderón de la Barca, Toledo, 14, 15 y 16 de enero de 2000, ed. Felipe B. Pedraza Jiménez, Rafael González Cañal y José Cano Navarro, Almagro, Universidad de Castilla-La Mancha, pp. 217-240.

- [2003]: «Francisco de Rojas Zorrilla», en Historia del Teatro Español, dir. Javier Huerta Calvo, Madrid, Gredos, I, pp. 1159-1189.

- [2003]: «Rojas Zorrilla y las comedias escritas en colaboración», en En torno al Teatro del Siglo de Oro. Jornadas XVI-XVII, ed. Olivia Navarro y Antonio Serrano, Almería, Institución de Estudios Almerienses, Diputación de Almería, pp. 29-43.

- [2004]: «Las comedias sobre el Gran Tamorlán de Persia», en Memoria de la palabra. Actas del VI Congreso de la Asociación Internacional Siglo de Oro. Burgos-La Rioja 15-19 de julio 2002, eds. María Luisa Lobato y Francisco Domínguez Matito, Madrid, Iberoamericana-Vervuert, pp. 917-928.

- [2005]: «Recursos espaciales del enredo en Rojas Zorrilla», en Espacio, tiempo y género en la comedia española. Actas de las II Jornadas de teatro clásico. Toledo, 14, 15 y 16 de noviembre de 2003, ed. Felipe B. Pedraza Jiménez, Rafael González Cañal y Gemma Gómez Rubio, Almagro, Universidad de Castilla-La Mancha, pp. 171-191.

— [2005]: «El rey Enrique el enfermo en el teatro español del Siglo de Oro», en Actas del Congreso "El Siglo de Oro en el nuevo milenio», ed. Carlos Mata y Miguel Zugasti, Pamplona, EUNSA, I, pp. 829-841.

- [2005]: «El espacio escénico en las comedias de capa y espada de Rojas Zorrilla», en Escenografía y escenificación en el teatro español del Siglo de Oro. Actas del II Curso sobre teoría y práctica del teatro, organizado por el Aula Biblioteca Mira de Amescua y el Centro de Formación Continua, celebrado en Granada (10-13 noviembre, 2004), ed. Roberto Castilla Pérez y Miguel González Dengra, Granada, Universidad de Granada, pp. 169-199.

- [2006]: «Rojas Zorrilla ante el entremés», en Edad de Oro Cantabrigense. Actas del VII Congreso de la Asociación Internacional del Siglo de Oro (AISO) (Robinson College, Cambridge, 18-22 de julio, 2005), ed. Anthony Close con la colaboración de Sandra $\mathrm{M}^{\mathrm{a}}$ Fernández Vales, Madrid, AISO, pp. 311-317.

- [2006]: «Rivalidades familiares en el teatro del Siglo de Oro: Los amantes de Verona», en El Siglo de Oro en escena. Homenaje a Marc Vitse, ed. Odette Gorsse y Frédéric Serralta, Toulouse, Presses Universitaires du Mirail-Consejería de Educación de la Embajada de España en Francia, pp. 405-418.

- [en prensa]: «Incógnitas y hallazgos en el repertorio dramático de Rojas Zorrilla», en Los segundones en el teatro español del Siglo de Oro, Gargnano di Garda, 1921 de septiembre de 2005.

- [en prensa]: «La transmisión impresa del teatro de Rojas Zorrilla», en Lectura y Signo, 2 (2007). 
- [2007]: «La poesía de un dramaturgo: poemas panegíricos y ocasionales de Rojas Zorrilla», en Teatro de palabras. Revista sobre teatro áureo, 1, pp. 47-65.

González CAÑal, Rafael, Ubaldo Cerezo Rubio y Germán Vega García-Luengos [2007]: Bibliografía de Francisco de Rojas Zorrilla, Kassel, Reichenberger.

GouldSON, Kathleen [1939]: A critical survey of the drama of Rojas Zorrilla, Tesis M.A., Liverpool (véase BHS, XLIX (1972), p. 332).

- [1939]: «Seventeenth-century Spain as Seen in the Drama of Rojas Zorrilla», en BSS, XVI, pp. 168-181.

- [1939]: «Religion and superstition in the plays of Rojas Zorrilla», en Bulletin of Hispanic Studies, XVI, pp. 168-181. Reeditado en Three Studies in Golden Age Drama (Spanish Golden Age Poetry and Drama), ed. E. Allison Peers, Liverpool, Institute of Hispanic Studies, 1946, pp. 89-101.

- [1940]: «Rojas Zorrilla and seventeenth-century Spain», en Bulletin of Hispanic Studies, 17, pp. 116-126. Reeditado en Three Studies in Golden Age Drama (Spanish Golden Age Poetry and Drama), ed. E. Allison Peers, Liverpool, Institute of Hispanic Studies, 1946, pp. 101-118.

GRANJA, Agustín de la [1993]: «Una carta con indicaciones escénicas para el autor de comedias Roque de Figueroa», en Revista Canadiense de Estudios Hispánicos (Toronto), XVII, n. ${ }^{\circ}$ 2, pp. 383-388.

- [2006]: «Comedias del Siglo de Oro censuradas por la Inquisición (Con noticia de un texto mal atribuido a Rojas Zorrilla)», en El Siglo de Oro en escena. Homenaje a Marc Vitse, ed. Odette Gorsse y Frédéric Serralta, Toulouse, Presses Universitaires du Mirail-Consejería de Educación de la Embajada de España en Francia, pp. 435-448.

HARTWELL, Ruth Hatch [1952]: The development of the 'comedia de figuron' in the seventeenth and eighteenth centuries, Albuquerque (New Mexico), Tesis doctoral inédita. Universidad de New Mexico.

Howe, Elizabeth Teresa [1985]: «Del rey abajo, ninguno and menosprecio de corte reconsidered», en Journal of Hispanic Philology, IX, pp. 133-146.

Julio, M. ${ }^{a}$ Teresa [1992]: «Planteamientos de la trama en el teatro de Rojas Zorrilla», en Anuario de Filología (Barcelona), XV, Sección F, n.o 3, pp. 59-72.

- [1996]: La recepción dramática: aplicación al teatro de Rojas Zorrilla, Kassel, Edition Reichenberger.

- [1996]: «La diégesis en la mímesis: el relato en el teatro de Rojas Zorrilla», en Studia Aurea. Actas del III congreso internacional de la AISO, vol. II, Pamplona-Toulouse, GRIMSO-LEMSO, pp. 197-204.

- [1998]: «La ocultación en la comedia de enredo de Rojas Zorrilla», en La comedia de enredo. Actas de las XX Jornadas de Teatro Clásico. Almagro, julio de 1997, ed. de Felipe B. Pedraza Jiménez y Rafael González Cañal, Almagro (Ciudad Real), Universidad de Castilla-La Mancha-Festival de Almagro, pp. 237-253.

- [1999]: «Obligados y ofendidos, una modélica comedia de capa y espada en busca de puesta en escena», en Doce comedias buscan un tablado. Cuadernos de Teatro Clásico, 11, dir. Felipe B. Pedraza Jiménez, Compañía Nacional de Teatro Clásico, pp. 191-217.

- [1999]: «Entre bobos anda el juego... y todos eran fulleros», en Boletín de la Compañía Nacional de Teatro Clásico, núm. 40, pp. 4-5.

- [2000]: «Hiperdramatismo en Rojas Zorrilla: ¿innovación o continuidad?», en Francisco de Rojas Zorrilla, poeta dramático. Actas de las XXII Jornadas de teatro clásico, Almagro 13, 14 y 15 de julio de 1999, ed. Felipe B. Pedraza Jiménez, Rafael González Cañal y Elena Marcello, Almagro (Ciudad Real), Universidad de CastillaLa Mancha-Festival de Almagro, pp. 179-208. 
- [2003]: «Amor y dolor en Rojas Zorrilla: dos caras de la misma moneda», en Toledo: entre Calderón y Rojas. IV Centenario del nacimiento de don Pedro Calderón de la Barca, Toledo, 14, 15 y 16 de enero de 2000, ed. Felipe B. Pedraza Jiménez, Rafael González Cañal y José Cano Navarro, Almagro, Universidad de Castilla-La Mancha, pp. 173-193.

- [2003]: «Tradición y creación en Los encantos de Medea de Francisco de Rojas Zorrilla», en Medeas. Versiones de un mito desde Grecia hasta hoy, ed. Aurora López y Andrés Pociña, Granada, Universidad de Granada, pp. 779-795.

- [2004]: «Francisco de Rojas Zorrilla: Un dramaturgo metido a poeta. Las fiestas de 1637», en Memoria de la palabra. Actas del VI Congreso de la Asociación Internacional Siglo de Oro. Burgos-La Rioja 15-19 de julio 2002, ed. María Luisa Lobato y Francisco Domínguez Matito, Madrid, Iberoamericana-Vervuert, pp. 1121-1132.

- [2004]: «Vicisitudes editoriales de una comedia áurea: Casarse por vengarse de Rojas Zorrilla», en La memoria de los libros. Estudios sobre la historia del escrito y de la lectura en Europa y en América, I, ed. M. Isabel de Páiz Hernández, Salamanca, Instituto de Historia del Libro y de la Lectura, pp. 627-637.

- [2004]: «Rojas Zorrilla y su atractivo mediático», en XIII Seminario Internacional: Teatro, Prensa y Nuevas Tecnologías, Madrid, Visor-Libros-UNED, pp. 373-386

- [2005]: «Tramoyas y artificios en Los encantos de Medea», en Espacio, tiempo y género en la comedia española. Actas de las II Jornadas de teatro clásico. Toledo, 14, 15 y 16 de noviembre de 2003, ed. Felipe B. Pedraza Jiménez, Rafael González Cañal y Gemma Gómez Rubio, Almagro, Universidad de Castilla-La Mancha, pp. 193-213.

- [2005]: «Rojas y el drama de honor: afinidades y disidencias calderonianas», en Actas del Congreso «El Siglo de Oro en el nuevo milenio», ed. Carlos Mata y Miguel Zugasti, Pamplona, EUNSA, II, pp. 921-929.

- [2005]: «La suerte escénica de Entre bobos anda el juego», en La maravilla escrita. Torquemada y el Siglo de Oro, León, Universidad de León, pp. 455-472.

- [2006]: «Crimen y castigo en el ámbito doméstico», Ínsula, n. ${ }^{\circ} 714$, junio 2006, pp. $15-17$

- [2006]: «La metateatralidad como recurso dramático en Casarse por vengarse», En torno al Teatro del Siglo de Oro, Jornadas XVIII-XX, Almería, Instituto de Estudios Almerienses, Diputación de Almería, pp. 59-74.

- [2007]: «Bruto, el loco cuerdo de Lucrecia y Tarquino de Rojas Zorrilla», en Locos, figurones y quijotes en el teatro de los Siglos de Oro. Actas selectas del XII Congreso de la Asociación Internacional de Teatro Español y Novohispano de los Siglos de Oro. Almagro, 15, 16 y 17 de julio de 2005, ed. Germán Vega GarcíaLuengos y Rafael González Cañal, Almagro, Universidad de Castilla-La Mancha, pp. 283-297.

- (ed.) [2007]: Academia burlesca que se hizo en Buen Retiro a la majestad de Filipo Cuarto el Grande. Año de 1637, Madrid, Iberoamericana Vervuert (Biblioteca Áurea Hispánica, 41).

- [en prensa]: «La vil Numancia vuestro honor profana: Cervantes y Rojas ante el drama numantino», en Actas del Congreso Internacional sobre "Cervantes y su tiempo», León, 2-5 noviembre de 2005.

- [en prensa]: «El arte de fingir: juegos metateatrales en Lucrecia y Tarquino», Lectura y Signo, 2 (2007).

KenNington, Nancy L. [1962]: Rojas Zorrilla and the 'comedia de figurón', Tesis M. A. inédita, Chapel Hill, University of North Carolina.

LANCASTER, H. C. [1917-1918]: «The ultimate source of Rotrou's Venceslas and of the Rojas Zorrilla's No hay ser padre siendo rey», en Modern Philology (Chicago), XV, pp. 435-440. 
LANOT, J. R. y ViTSE, M. [1976] : «Eléments pour une théorie du figuron», en Caravelle. Cahiers du monde hispanique et luso-brésilien, 27, pp. 189-213.

Lara Alberola, Eva [2005]: «Pervivencia de la hechicera clásica en el teatro español barroco», en La recepción de los clásicos, ed. Rafael Beltrán Llavador, Purificación Ribes Traver y Jorge L. Sanchis Llopis, número monográfico de Quaderns de Filologia, Valencia, X, pp. 169-184.

LARSON, Catherine [1991]: «Grice's Maxiums and Rojas Zorrilla's Entre bobos anda el juego», en Language and the Comedia: Theory and Practice, Lewisburg, PA, Bucknell UP.

LEYTE VIDAL, Nocidia J. [1977]: El personaje 'gracioso' en la dramaturgia de Francisco de Rojas Zorrilla, Tesis doctoral inédita, Catholic University of America.

MACCURDY, Raymond R. [1954]: «A note on Rojas Zorrilla's gracioso 'Guardainfante'», en Bulletin of the Comediantes, VI, pp. 1-4.

- [1956]: «More on 'The gracioso takes the audience into his confidence': the case of Rojas Zorrilla», en Bulletin of the Comediantes, VIII, pp. 15-16.

- [1957]: «Francisco de Rojas Zorrilla», en Bulletin of the Comediantes, IX, pp. 7-9.

- [1958]: Francisco de Rojas Zorrilla and the tragedy, Albuquerque, University of New Mexico Press.

- [1959]: «The bathing nude in Golden Age drama», en Romance Notes (Chapel Hill), I, núm. 1, pp. 36-39.

- [1960]: «The Numantia Plays of Cervantes and Rojas Zorrilla. The shift from collective to personal tragedy», en Symposium (Syracuse), XIV, pp. 100-120.

- [1965]: Rojas Zorrilla: Bibliografía crítica, Madrid, Consejo Superior de Investigaciones Científicas (Cuadernos Bibliográficos, XVIII).

- [1966]: «Two instances of Rojas Zorrilla's Parody of Spanish Ballads», en Homenaje a Rodríguez Moñino. Estudios de erudición que le ofrecen sus amigos o discípulos hispanistas norteamericanos, I, Madrid, Castalia, pp. 367-370.

- [1966]: «The Spanish Sources of Paul Scarron's Jodelet duelliste», en Hispanic Studies in honor of Nicholson B. Adams, Chapel Hill, University of Carolina Studies in the Romance Languages and Literature, 59.

- [1968]: Francisco de Rojas Zorrilla, Nueva York, Twayne Publishers, Inc. (Fragmentos reproducidos en Francisco Rico, dir., Historia y crítica de la Literatura Española, 3, Bruce W. Wardropper, Siglos de Oro: Barroco, Barcelona, Crítica, 1983, pp. 893-899).

- [1974]: «On the use of Rape of Lucrecia», en Estudios literarios de hispanistas norteamericanos dedicados a H. Hatzfeld con motivo de su 800 aniversario, Barcelona, pp. 297-308.

- [1979]: «Women and Sexual Love in the Plays of Rojas Zorrilla: Tradition and Innovation», en Hispania (California), LXII, pp. 255-265.

- [1979] «Rojas Zorrilla's gracioso and the Renunciation of Honor», en Studies in Honor of Gerald E. Wade, Madrid, Porrúa Turanzas, pp. 167-177.

MACKENZIE, Ann L. [1976]: «Examen de El monstruo de la fortuna: comedia compuesta por Calderón (I), Pérez de Montalbán (II) y Rojas Zorrilla (III)», en Hacia Calderón. Tercer Coloquio Anglogermano. Londres 1973, Berlin /Nueva York, Gualter de Gruyter, pp. 110-125.

- [1982]: «A Study in Dramatic Contrasts. The Siege of Antwerp in A Larum for London and El saco de Amberes», en Bulletin of Hispanic Studies, LIX, pp. 383-300.

- [1983]: «Vélez de Guevara as Dramatic Collaborator, with specific Reference to También la afrenta es veneno (I. Vélez, II. Coello, III. Rojas Zorrilla)», en Antigüedad y Actualidad de Luis Vélez de Guevara. Estudios críticos, ed. C. George Peale et al., Amsterdam/Philadelphia, John Benjamins Publishing Co., pp. 182-202. 
- [1983]: «El saco de Amberes, comedia falsamente atribuida a Calderón. )Es de Rojas Zorrilla?», en Hacia Calderón. Sexto Coloquio Anglogermano (Würzburg, 1981), publicado por Hans Flasche y corredactado por Pedro Jaun-Tous, Franz Steiner Verlag GMBH, Wiesbaden, 1983, pp. 151-168.

- [1993]: La escuela de Calderón: estudio e investigación, Liverpool, Liverpool University Press.

- [1994]: Francisco de Rojas Zorrilla y Agustín Moreto. Análisis, Liverpool, Liverpool University Press.

Madroñal DurÁn, Abraham [2001]: «Lenguaje e historia: El Pleito que tuvo el diablo con el cura de Madrileños», en La teatralización de la historia en el Siglo de Oro español. Actas del III Coloquio del Aula-Biblioteca «Mira de Amescua» celebrado en Granada del 5 al 7 de noviembre de 1999 y cuatro estudios clásicos sobre el tema, ed. Roberto Castilla Pérez y Miguel González Dengra, Granada, Universidad de Granada, pp. 329-338.

MARCELlo, Elena E. [1998]: «Appunti sulla fortuna del teatro spagnolo in Italia: Il maritarsi per vendetta di G.A. Cicognini», en El teatro italiano. Actas del VII Congreso Nacional de Italianistas (21 al 23 de octubre de 1996), Valencia, pp. 399-406.

- [en prensa]: «La recepción del teatro de Francisco de Rojas Zorrilla en Italia. Algunas anotaciones», Lectura y Signo, 2 (2007).

- [en prensa]: «La furia celosa de Rodamonte en Lope y Rojas», en La pasión teatral de los celos en el Siglo de Oro. Actas del III Curso sobre teoría y práctica del teatro, organizado por el Aula-Biblioteca Mira de Amescua y Centro de Formación Continua celebrado en Granada (8-11 noviembre, 2006), ed. Remedios Morales Raya y Miguel González Dengra, Granada, Universidad de Granada.

Maroto CAmino, Mercedes [1997]: "'Ya no es Lucrecia, Lucrecia': Woman and 'limpieza de sangre' in Rojas Zorrilla's Lucrecia y Tarquino», en Revista Canadiense de Estudios Hispánicos, XXI, n. ${ }^{\circ}$ 2, pp. 329-351.

MARTíneZ VIDAL, Enrique [1984]: «Katharsis and Comic Relief in Rojas Zorrilla's Progne y Filomena», en Homenaje a Josep María Solá-Solé, ed. de Antonio TorresAlcalá et al., II, Barcelona, Puvill, pp. 85-90.

MCKENDRICK, Melveena [1994]: «Francisco de Rojas Zorrilla (1607-1648)», en El teatro en España (1490-1700), Palma de Mallorca, Olañeta, pp. 181-187.

McVAY, Ted E. Jr. [1993]: «Loss, Language and Politics in Two Golden Age Works: The Progne y Filomena Plays of Guillén de Castro y Francisco de Rojas Zorrilla», en Looking at the Comedias Symposium on Golden Age Drama, Lanham M. D. University Press of America, pp. 141-147.

- [1994]: «Sebastián y Latre=s refundición of Rojas Zorrilla=s Progne y Filomena as a reflection of social change», en Selected Proceedings: Lousiana Conference on Hispanic Languages and Literatures 1994, ed. Joseph V. Ricapito, Baton Rouge, LA, Lousiana State UP, pp. 161-168.

MÉGEVAND, Sophie [1988]: «Un phénomène original: Trois adaptations simultanées d=une pièce espagnole», en Colloquium Helveticum, 8, pp. 61-80.

MeSOnero Romanos, Ramón de [1861]: «Apuntes biográficos, bibliográficos y críticos de Don Francisco de Rojas Zorrilla», en F. de Rojas Zorrilla, Comedias Escogidas, Madrid, M. Rivadeneyra (BAE, LIV), pp. V-XXIV.

Miguel Reboles, M. Teresa de [2003]: «Algunas consideraciones hebraicas en Rojas y Calderón», en Toledo: entre Calderón y Rojas. IV Centenario del nacimiento de don Pedro Calderón de la Barca, Toledo, 14, 15 y 16 de enero de 2000, ed. Felipe B. Pedraza Jiménez, Rafael González Cañal y José Cano Navarro, Almagro, Universidad de Castilla-La Mancha, pp. 111-123. 
MILEgO, Julio [1909]: El teatro en Toledo durante los siglos XVI y XVII, Valencia, Tip. de Manuel Pau, cap. XI.

MoIR, Duncan [1973]: «Notes on the significance and text of Rojas Zorrilla's Cada cual lo que le toca», en Studies in Spanish Literature of the Golden Age presented to Edward M. Wilson, Londres, Tamesis Books, pp. 149-159.

NAGY, Edward [1987]: «El soldado y el calabozo. Las intercalaciones picarescoentremesiles de Luis Vélez de Guevara», en Iberoromania, 25, pp. 38-47 (Sobre El catalán Serrallonga).

NAVARro Durán, Rosa [2003]: «Mecanismos de enredo en comedias de Rojas», en Toledo: entre Calderón y Rojas. IV Centenario del nacimiento de don Pedro Calderón de la Barca, Toledo, 14, 15 y 16 de enero de 2000, ed. Felipe B. Pedraza Jiménez, Rafael González Cañal y José Cano Navarro, Almagro, Universidad de Castilla-La Mancha, pp. 155-171.

Nougué, A. [1968]: «Fernán González en el teatro español», en Boletín de la Institución Fernán González, XLVI, pp. 107-116 y 246-258.

NowAK, Lisa J. [2005]: «Not so 'Happily ever after': Equivocal Ideology in Plays by Calderón, Hurtado de Mendoza, and Rojas Zorrilla», en Hispanófila, Chapel Hill, NC, núm. 144, pp. 33-44.

ORTigOZA Vieyra, Carlos [1954]: Los móviles de la «comedia» en Lope, Alarcón-TirsoMoreto-Rojas-Calderón, México, Universidad Nacional de México.

- [1957]: «Del rey abajo, ninguno, de Rojas Zorrilla, estudiada a través de sus móviles», en Bulletin of the Comediantes, IX, pp. 1-4.

PACHeco Y COSTA, Alejandra [2000]: «Música y teatro en Rojas Zorrilla. Lo que quería ver el marqués de Villena», en Francisco de Rojas Zorrilla, poeta dramático. Actas de las XXII Jornadas de teatro clásico, Almagro 13, 14 y 15 de julio de 1999, ed. Felipe B. Pedraza Jiménez, Rafael González Cañal y Elena Marcello, Almagro (Ciudad Real), Universidad de Castilla-La Mancha-Festival de Almagro, pp. 271-300.

PALACIOS FERnÁNDEZ, Emilio [2000]: «Pervivencias del teatro barroco. Recepción de Rojas Zorrilla en el siglo XVIII», en Francisco de Rojas Zorrilla, poeta dramático. Actas de las XXII Jornadas de teatro clásico, Almagro 13, 14 y 15 de julio de 1999, ed. Felipe B. Pedraza Jiménez, Rafael González Cañal y Elena Marcello, Almagro (Ciudad Real), Universidad de Castilla-La Mancha-Festival de Almagro, pp. 349-378.

PARDo CANAlís, Enrique [1963]: «Ruiz de Alarcón-Rojas Zorrilla-Moreto», número especial de la Revista de ideas estéticas, XXI, pp. 259-285.

PASTENA, Enrico di [2000]: «Sin honra no hay amistad y El desdén, con el desdén: aspectos del teatro cómico de F. de Rojas y A. Moreto», en Francisco de Rojas Zorrilla, poeta dramático. Actas de las XXII Jornadas de teatro clásico, Almagro 13, 14 y 15 de julio de 1999, ed. Felipe B. Pedraza Jiménez, Rafael González Cañal y Elena Marcello, Almagro (Ciudad Real), Universidad de Castilla-La ManchaFestival de Almagro, pp. 103-120.

PASTOR COMín, Juan José [2000]: «La música como recurso dramático en la obra de Rojas Zorrilla», en Francisco de Rojas Zorrilla, poeta dramática. Actas de las XXII Jornadas de teatro clásico, Almagro 13, 14 y 15 de julio de 1999, ed. Felipe B. Pedraza Jiménez, Rafael González Cañal y Elena Marcello, Almagro (Ciudad Real), Universidad de Castilla-La Mancha-Festival de Almagro, pp. 243-270.

- [1999]: «La comedia de enredo: Abre el ojo de Francisco de Rojas Zorrilla y El amor al uso de Antonio de Solís. Análisis comparativo», en Epos, XV, pp. 149-174.

- [2001]: «Abre el ojo y El amor al uso: marcas de género y configuración del 'enredo' en Rojas Zorrilla y Antonio de Solís», en Actas del V Congreso de la Asociación Internacional Siglo de Oro, Munster 1999, ed. Christoph Strosetzki, Vervuert, Iberoamericana, pp. 964-987. 
PAVesio, Monica [2004]: «L'onore spagnolo nel teatrofrancese secentesco: il caso singolare del triplice adattamento teatrale di Obligados y ofendidos y gorrón de Salamanca di Rojas Zorrilla (Boisrobert, Scarron, Thomas Corneille)», en Il tema dell'onore nel Teatro Barocco in Europa. Atti del convegno internazionale (Losanna, 14-16 novembre 2002), a cura di Alberto Roncaccia, Massimiliano Spiga e Antonio Stäuble, Firenze, Franco Cesati Editore, pp. 203-218.

PEDRAZA JimÉneZ, Felipe B. [1999]: «Rojas en el clásico», prólogo a la edición de Entre bobos anda el juego, de Francisco de Rojas Zorrilla, Madrid, Compañía Nacional de Teatro Clásico, pp. [5]-[8].

- [2000]: «Francisco de Rojas Zorrilla, poeta cómico», en F. de Rojas Zorrilla, Obligados y ofendidos, Madrid, RESAD-Ed. Fundamentos, pp. 37-63; nueva versión [2003], en Toledo: entre Calderón y Rojas. IV Centenario del nacimiento de don Pedro Calderón de la Barca, Toledo, 14, 15 y 16 de enero de 2000, ed. Felipe B. Pedraza Jiménez, Rafael González Cañal y José Cano Navarro, Almagro, Universidad de Castilla-La Mancha, pp. 155-171.

- [2003]: «Los lugares imaginarios en Rojas Zorrilla: Persiles y Sigismunda», en Loca ficta. Los espacios de la maravilla en la Edad Media y Siglo de Oro, ed. Ignacio Arellano Ayuso, Madrid, Iberoamericana, Vervuert, pp. 333-348.

- [2003]: «Variantes del galanteo en Rojas Zorrilla», en Ronda, cortejo y galanteo en el teatro español del Siglo de Oro. Actas del I Curso sobre teoría y práctica del teatro, organizado por el Aula-Biblioteca Mira de Amescua y el Centro de Formación Continua, celebrado en Granada, (7-9 noviembre, 2002), ed. Roberto Castilla Pérez, Granada, Universidad de Granada, pp. 113-133.

- [2003]: «El jardín de Falerina: y la recreación escénica de las caballerías», en Giornate Calderoniane, Calderón 2000. Atti del Convegno Internazionale Palermo 14-17 Dicembre 2000, a cura di Enrica Cancelliere, Palermo, Flaccovio Editore, pp. 171-185.

- [2003]: «Abrir el ojo bajo el antiguo régimen», en $>$ Estaba el jardín en flor...= Homenaje a Stefano Arata, Criticón, 87, 88 y 89, pp. 637-648.

- [2004]: «La comedia española como yuxtaposición de estilos. El caso de Rojas Zorrilla», en Edad de Oro, XXIII, pp. 339-354.

- [2004]: «Rojas Zorrilla ante la figura del donaire», en Congreso Internacional Proyección y significados del teatro clásico español, Madrid, mayo de 2003, coord. José María Díez Borque y José Alcalá-Zamora, Madrid, Sociedad Estatal para la Acción Cultural Exterior, pp. 287-306.

- [2004]: «Los ingredientes trágicos del enredo cómico. El caso de Rojas Zorrilla», en Charisterion. Francisco Martín Garcia oblatum, coord. Ignacio García Pinilla y Santiago Talavera Cuesta, Cuenca, Ediciones de la Universidad de Castilla-La Mancha, pp. 753-775.

- [2005]: «De Rojas Zorrilla a Enciso Castrillón: Abrir el ojo en la escena decimonónica», en A zaga de tu huella. Homenaje al prof. Cristóbal Cuevas, Málaga, Universidad de Málaga, I, pp. 539-557.

- [2005]: «A vueltas con la taxonomía: La traición busca el castigo de Rojas Zorrilla», en Espacio, tiempo y género en la comedia española. Actas de las II Jornadas de teatro clásico. Toledo, 14, 15 y 16 de noviembre de 2003, ed. Felipe B. Pedraza Jiménez, Rafael González Cañal y Gemma Gómez Rubio, Almagro, Universidad de Castilla-La Mancha, pp. 453-483.

- [2005]: «Rojas Zorrilla: un teatro para los oídos», en Escenografía y escenificación en el teatro español del Siglo de Oro. Actas del II Curso sobre teoría y práctica del teatro, organizado por el Aula Biblioteca Mira de Amescua y el Centro de Forma- 
ción Continua, celebrado en Granada (10-13 noviembre, 2004), ed. Roberto Castilla Pérez y Miguel González Dengra, Granada, Universidad de Granada, pp. 403-421.

- [2005]: «De Tirso a Rojas Zorrilla. Algunas notas sobre las piezas cómicas», en Ramillete de gustos: Burlas y veras en Tirso de Molina, ed. Ignacio Arellano, Segovia, Fundación Instituto Castellano y Leonés de la Lengua (col. Beltenebros, n. $\left.{ }^{\circ} 10\right)$, pp. 357-371.

- [2005]: «Rojas Zorrilla ante la comedia de santos: Santa Isabel, reina de Portugal», en Homenaje a Henri Guerreiro. La hagiografía entre historia y literatura en la España de la Edad Media y del Siglo de Oro, ed. Marc Vitse, Pamplona, Universidad de Navarra-Iberoamericana Vervuert, pp. 967-983.

- [2006]: «Humor y comicidad en Rojas Zorrilla: de la caricatura al esperpento», en Demócrito áureo. Los códigos de la risa en el Siglo de Oro, ed. Ignacio Arellano y Victoriano Roncero, Sevilla, Renacimiento, pp. 179-211.

- [2006]: «Un dramaturgo barroco ante el exotismo: Francisco de Rojas Zorrilla», en Le arti della scena e l'esotismo in età moderna, a cura di Francesco Cotticelli e Paologiovanni Maione, Napoli, Turchini Edizioni, pp. 81-93.

- [2006]: «Donde hay agravios no hay celos: un éxito olvidado», En torno al Teatro del Siglo de Oro, Jornadas XVIII-XX, Almería, Instituto de Estudios Almerienses, Diputación de Almería, pp. 145-168.

- [2007]: Estudios sobre Rojas Zorrilla (ante el IV centenario), Almagro, Universidad de Castilla-La Mancha (col. «Corral de Comedias», 21).

- [2006]: «Cada cual lo que le toca. Notas de lectura», en El Siglo de Oro en escena. Homenaje a Marc Vitse, ed. Odette Gorsse y Frédéric Serralta, Toulouse, Presses Universitaires du Mirail-Consejería de Educación de la Embajada de España en Francia, pp. 719-731.

- [en prensa]: «Personas reales y justicia poética: Lope, Guillén de Castro, Rojas Zorrilla», Lectura y signo, 2 (2007).

PedraZa JiméneZ, Felipe B., Rafael GonZÁlez CaÑal y Elena Marcello, eds. [2000]: Francisco de Rojas Zorrilla, poeta dramático. Actas de las XXII Jornadas de teatro clásico, Almagro 13, 14 y 15 de julio de 1999, Almagro (Ciudad Real), Universidad de Castilla-La Mancha-Festival de Almagro.

Peláez Martín, Andrés [2000]: «Francisco de Rojas, un ausente en la escena», en Francisco de Rojas Zorrilla, poeta dramático. Actas de las XXII Jornadas de teatro clásico, Almagro, 13, 14 y 15 de julio de 1999, ed. Felipe B. Pedraza Jiménez, Rafael González Cañal y Elena Marcello, Almagro, Universidad de Castilla-La ManchaFestival de Almagro, pp. 395-403.

Place, Edwin B. [1939]: «Notes on the grotesque: the 'comedia de figurón'», en Publications of the Modern Language Association (Nueva York), LIV, pp. 412-421. PociÑA, Andrés [1996]: «Tres dramatizaciones del tema de Medea en el Siglo de Oro español: Lope de Vega, Calderón de la Barca y Rojas Zorrilla», en Pervivencia y actualidad de la cultura clásica, ed. J.M. González y A. Pociña Pérez, Granada, Universidad de Granada, pp. 287-314.

POWERS, Harriet. B. [1971]: «The grotesque vision of Rojas Zorrilla», en Bulletin of the Comediantes, 23, pp. 1-6.

PROFETI, María Gracia [1990]: «Francisco de Rojas Zorrilla (1607-1648)», en VV. AA., Historia de la literatura española I. Desde los orígenes al siglo XVII, Madrid, Cátedra, pp. 650-651.

- [2003]: «Espacio geográfico y signo teatral: Toledo en el teatro de Rojas Zorrilla», en Toledo: entre Calderón y Rojas. IV Centenario del nacimiento de don Pedro Calderón de la Barca, Toledo, 14, 15 y 16 de enero de 2000, ed. Felipe B. Pedraza 
Jiménez, Rafael González Cañal y José Cano Navarro, Almagro, Universidad de Castilla-La Mancha, pp. 127-153.

REgueiro, José M. [1995]: «Textual discontinuities and the problems of closure in the Spanish Drama of the Golden Age», en Cultural Authority in Golden Age Spain, ed. Marina S. Brownlee y Hans Ulrich Grumbrecht, Parallax, Re-Visions of Culture and Society, Baltimore, MD, Johns Hopkins UP, pp. 28-50.

REICHENBERGER, Arnold G. [1959]: «Rojas Zorrilla's Del rey abajo, ninguno as a Spätcomedia», en Stil und Formprobleme in der Literatur, Vorträge des VII Kongresses der Internationalen Vereinigung für moderne Sprachen und Literaturen in Heidelberg, Heidelberg, Carl Winter, pp. 194-200.

- [1964]: «Recent Publications concerning Francisco de Rojas Zorrilla», en Hispanic Review (Filadelfia), XXXII, pp. 351-359 (Bibliografía).

Reyes Palacios, Felipe [2005]: «Lo que son mujeres, comedia de figurón de Francisco de Rojas refundida por Gorostiza», en Estudios de teatro español y novohispano. Asociación Internacional de Teatro Español y Novohispano de los Siglos de Oro, ed. Melchora Romanos, Ximena González y Florencia Calvo, Buenos Aires, Universidad de Buenos Aires, pp. 467-484.

RodrígueZ CÁCERES, Milagros [1999]: «Perfil biográfico y literario de Francisco de Rojas Zorrilla», en Boletín de la Compañía Nacional de Teatro Clásico, núm. 40, pp. 1-3.

- [2006]: «La transmisión textual de Donde hay agravios no hay celos de Francisco de Rojas Zorrilla», en Edad de Oro Cantabrigense. Actas del VII Congreso de la Asociación Internacional del Siglo de Oro (AISO) (Robinson College, Cambridge, 18-22 de julio, 2005), ed. Anthony Close con la colaboración de Sandra Ma Fernández Vales, Madrid, AISO, pp. 527-532.

RodrígueZ PUÉRTOLAS, Julio [1967]: «Alienación y realidad en Rojas Zorrilla», en Bulletin Hispanique, LXIX, pp. 325-346. (Reimpreso en De la Edad Media a la edad conflictiva, Madrid, Gredos, 1972, pp. 339-363).

RoIG, Adrien [1991]: «Une manifestation de catalanophilie littéraire: El catalán Serrallonga. Comedia de tres ingenios», en Melanges de la Casa de Velázquez, XXVI, n. ${ }^{\circ}$ 2, pp. 153-170.

- [1992]: «Una manifestación de catalanofilia literaria: El catalán Serrallonga, comedia de tres ingenios», en Actas del X Congreso de la Asociación Internacional de Hispanistas. Barcelona, 21-26 de agosto de 1989, ed. Antonio Vilanova, Barcelona, PPU, pp. 1053-1066.

Rubio SAn Román, Alejandro y Elena Martínez CARro [2006]: «Aportación documental a la obra de Rojas Zorrilla», en Dicenda, 24, pp. 219-234.

RundLE, James U. [1950]: «D'Avenant's The Man's the Master and the Spanish Source», en Modern Language Notes (Baltimore), LXV, pp. 194-196.

SAlvador Plans, Antonio [1992]: «Nuestra Señora de Atocha de Rojas Zorrilla», en La 'fabla antigua' en los dramaturgos del siglo de oro, Cáceres, Universidad de Extremadura, pp. 135-136.

SCARAmuZZA VidOni, Mariarosa [2000]: «Peripezie di Persiles e Sigismunda sul palcoscenico: l'adattamento teatrale di Rojas Zorrilla», en Atti del Convegno di Studi Napoli, 22-24 aprile 1999, Drammaturgia e spettacolarità nel teatro iberico dei Secoli d'Oro, Napoli, Edizione del Paguro, pp. 203-216.

SchmidT, Gisela [1959]: Studien zu den Komödien des Don Francisco de Rojas Zorrilla, Elbing, Tesis doctoral, Universidad de Colonia.

SERRANO GARCíA, Virtudes [1974]: «La función de la mujer en tres dramas de honor del siglo XVII», en Estudios Literarios dedicados al profesor Mariano Baquero Goyanes, Murcia, pp. 495-510 (sobre Casarse por vengarse). 
Shergold, N. D. y John E. VAREY [1964]: «A problem in the staging of autos sacramentales in Madrid (1647-1648)», en Hispanic Review, XXXII, pp. 12-35 (sobre la representación en 1647 del auto de Rojas El gran patio de palacio).

SímINI, Diego [1996]: «Casarse por vengarse di Rojas Zorrilla nella traduzione di Giacinto Andrea Cicognini: Maritarsi per vendetta», en M. G. Profeti et al., Tradurre, riscrivere, mettere in scena, Firenze, Alinea Editrice, pp. 95-116.

- [en prensa]: «Celos cruzados en Casarse por vengarse de Rojas Zorrilla», en La pasión teatral de los celos en el Siglo de Oro. Actas del III Curso sobre teoría y práctica del teatro, organizado por el Aula-Biblioteca Mira de Amescua y Centro de Formación Continua celebrado en Granada (8-11 noviembre, 2006), ed. Remedios Morales Raya y Miguel González Dengra, Granada, Universidad de Granada.

SolA-SolÉ, Josep M. y Montserrat D. [1972]: «Los Mahomas de Rojas Zorrilla», en Revista de Estudios Hispánicos (Alabama), VI, pp. 13-18.

Stoll, Anita K. [1996]: «La gran comedia de La Baltasara by 'tres ingenios de la corte'», en Bulletin of the Comediantes, 48, núm. 2, pp. 329-338.

- [1998]: «Francisco de Rojas Zorrilla (1607-1648)», en Spanish Dramatists of the Golden Age. A Bio-Bibliographical Sourcebook, ed. Mary Parker, Westport, London, Greenwood Press, pp. 177-187.

SuÁREZ MirAmón, Ana [1993]: «La función cómica del personaje Guardainfante en el teatro de Rojas», en Ex-Libris. Homenaje al profesor Fradejas Lebrero, Madrid, Universidad Nacional de Educación a Distancia, I, pp. 453-467.

- [1996]: «Magia y elogio del intelecto: Lo que quería ver el marqués de Villena», en Studia Aurea. Actas del III congreso internacional de la AISO (Toulouse, 1993), vol. II, Pamplona-Toulouse, GRISO-LEMSO, II, pp. 373-384.

- [1996]: «Itinerarios madrileños en Rojas Zorrilla», en Caminería Hispánica. Actas del III Congreso..., Guadalajara, AACHE Ediciones, III, pp. 339-363.

- [2004]: «La profanización del mito de Venus Anadiomena en Rojas y Calderón», en Memoria de la palabra. Actas del VI Congreso de la Asociación Internacional Siglo de Oro. Burgos-La Rioja 15-19 de julio 2002, eds. María Luisa Lobato y Francisco Domínguez Matito, Madrid, Iberoamericana-Vervuert, pp. 1707-1716.

- [2005]: «El gran patio de palacio: ¿tercer auto de una trilogía del mundo?», en Actas del Congreso «El Siglo de Oro en el nuevo milenio», ed. Carlos Mata y Miguel Zugasti, Pamplona, EUNSA, II, pp. 1591-1603.

- [2005]: «La función del monólogo narrativo en Rojas Zorrilla», en Filología y lingüística: Estudios ofrecidos a Antonio Quilis, Madrid, CSIC-UNED-Universidad de Valladolid, II, pp. 2131-2155.

TESTAS, Jean [1965]: «À propos de la comedia Del rey abajo, ninguno», en Les Langues Neolatines, LIX, 172, pp. 34-40.

- [1975]: «Le féminisme de Francisco de Rojas Zorrilla», en Mélanges offerts à Charles Vincen Aubrun, ed. H. Vidal Sephiha, París, Éditions Hispaniques, II, pp. 302-322.

TORReS, José C. [1983]: «El léxico taurino en el ciclo teatral de Calderón», en Calderón. Actas del 'Congreso Internacional sobre Calderón y el teatro español del Siglo de Oro' (Madrid, 8-13 de junio de 1981), ed. Luciano García Lorenzo, Madrid, CSIC, II, pp. 1172-1219.

Trambaioli, Marcella [1995]: «Los encantos de Medea, de Rojas Zorrilla y la espectacularidad de la comedia de tramoya», en Texto y Espectáculo, ed. J. L. Suárez García, El Paso, University of Texas, pp. 107-116.

- [1996]: «Una obra mitológica de corral: Progne y Filomena de Rojas Zorrilla», en Bulletin of the Comediantes, Chapel Hill, XLVIII, núm. 2, pp. 275-294.

- [1997]: «Una obra mitológica de corral: Progne y Filomena de Rojas Zorrilla», en La década de oro de la comedia española: 1630-1640. Actas de las XIX Jornadas 
de teatro clásico. Almagro, julio de 1996, ed. Felipe B. Pedraza Jiménez y Rafael González Cañal, Almagro, Universidad de Castilla-La Mancha-Festival de Almagro, pp. 263-280.

Ullman, Pierre [1966]: «A theme of Del rey abajo ninguno and its analogy with Limpieza de sangre», en The Romanic Rewiew (Nueva York), LVII, pp. 25-34.

URBINA, Eduardo [1984]: «Eclipse real: sol y sombra en Del rey abajo, ninguno», en Bulletin of the Comediantes (Toronto), XXXVI, pp. 101-110.

VAlbuena PRAT, Ángel [1930]: «El ciclo dramático de Calderón», en Literatura dramática española, Barcelona, Labor, pp. 249-260.

- [1969]: «La fuerza trágica y cómica de Rojas Zorrilla», en El teatro español en su Siglo de Oro, Barcelona, Planeta, pp. 364-377.

VALLE ABAD, Federico del [1946]: «Rojas Zorrilla y Rotrou», en Influencia Española en La Literatura francesa. Ensayo crítico sobre Juan Rotrou (1609-1650), Ávila, Tipografía y Encuadernación de Senén Martín, pp. 218-240.

VAN ANTWERP, Margaret A. [1979]: «'El fénix es'. The symbolic structure of Del rey abajo ninguno», en Hispanic Review, XLVII, pp. 441-454.

Vega García-Luengos, Germán [1994]: «Treinta comedias desconocidas de Ruiz de Alarcón, Mira de Amescua, Vélez de Guevara, Rojas Zorrilla y otros de los mejores ingenios de España», en Criticón, 62, pp. 57-78.

- [2000]: «Más vale maña que fuerza: los enredos albaneses de una comedia atribuida a Rojas Zorrilla», en Francisco de Rojas Zorrilla, poeta dramático. Actas de las XXII Jornadas de teatro clásico, Almagro, 13, 14 y 15 de julio de 1999, ed. Felipe B. Pedraza Jiménez, Rafael González Cañal y Elena Marcello, Almagro (Ciudad Real), Universidad de Castilla-La Mancha-Festival de Almagro, pp. 55-88.

VILA, Juan Diego [2005]: «La abyección a escena: Dramaturgia e ideología en Cada cual lo que le toca de Rojas Zorrilla», en Estudios de teatro español y novohispano. Asociación Internacional de Teatro Español y Novohispano de los Siglos de Oro, ed. Melchora Romanos, Ximena González y Florencia Calvo, Buenos Aires, Universidad de Buenos Aires, pp. 485-496.

VILLARINO, Marta y FIADINO, Graciela [2000]: «Nuevas formas de la comicidad en Abre el ojo», en Francisco de Rojas Zorrilla, poeta dramático. Actas de las XXII Jornadas de teatro clásico, Almagro, 13, 14 y 15 de julio de 1999, ed. Felipe B. Pedraza Jiménez, Rafael González Cañal y Elena Marcello, Almagro (Ciudad Real), Universidad de Castilla-La Mancha-Festival de Almagro, pp. 89-102.

VolPE, Germana [2003]: «Per una edizione critica di El monstruo de la fortuna. La lavandera de Nápoles. Felipe Catanea», en Annali dell'Istituto Universitario Orientale. Sezione Romanza, 45:1, pp. 203-214.

- [2004]: «El monstruo de la fortuna, tra storia e finzione», en Annali dell'Istituto Universitario Orientale. Sezione Romanza, 46:1, pp. 227-236.

Walthaus, Rina [1998]: «Dos tragedias de tema clásico de Francisco de Rojas Zorrilla: Progne y Filomena y Lucrecia y Tarquino», en Teatro español del Siglo de Oro. Teoría y práctica, ed. Christoph Strosetzki, Frankfurt am Main-Madrid, VervuertIberoamericana, pp. 370-381.

- [1998]: «Mujeres en el teatro de tema clásico de Francisco de Rojas Zorrilla: entre tradicionalismo y subversión», en Las mujeres en la sociedad española del Siglo de Oro: ficción teatral y realidad histórica, eds. Juan Antonio Martínez Berbel y Roberto Castilla Pérez, Granada, Universidad de Granada, pp. 137-157.

WARDROPPER, Bruce W. [1961]: «The poetic world of Rojas Zorrilla's Del rey abajo, ninguno», en The Romanic Review (Nueva York), LII, pp. 161-172.

- [1997]: «De Lope de Vega a Calderón: Las aventuras milagrosas de don Juan de Castro», en Negotiating Past and Present: Studies in Spanish Literature for Javier Herrero, ed. David Thatcher Gies, Charlottesville, Rokwood Press, pp. 153-160. 
WeLleS, Marcia [1991]: «Rojas Zorrilla's Lucrecia y Tarquino: 'The Taming of Lucretia': Or, the Staging of Perverse Desire», en Romance Languages Annual, 3, pp. 622-629.

WhitAKer, Shirley B. [1983]: «La Baltasara in Perfomance, 1634-35: Repports from the Tuscan Embassy», en Antigüedad y actualidad de Luis Vélez de Guevara: estudios críticos, ed. C. George Peale, Amsterdam / Philadelphia, John Benjamins Publishings Company, pp. 203-206.

Whitby, William M. [1959]: «Appearance and reality in Del rey abajo, ninguno», en Hispania (Baltimore), XLII, pp. 186-191.

White, Ralph E. [1949]: «The social and economic background of the comedia de figurón», en The Centenary Review (Shreveport, Louisiana), I, pp. 46-51.

Wiltrout, Anne E. [1989]: «The comedia as Pastiche. Rojas Zorrilla's Lo que quería ver el marqués de Villena», en Los hallazgos de la lectura: estudios dedicados a Miguel Enguídanos, ed. de John Crispin, Enrique Pupo-Walker y Luis Lorenzo Rivero, Madrid, Porrúa Turanzas, pp. 49-61.

WiTTMAN, Brigitte [1962]: «Falscher lorbeer für Rojas Zorrilla?», en Ibero-Romania (Munich), I, pp. 261-268. 